\title{
A New Undergraduate Laboratory Course in Magnetic Design
}

\author{
Taufik Taufik, Scott McClusky, Jonathan Paolucci and Dale S. Dolan
}

\begin{abstract}
This paper presents a set of new laboratory experiments developed in conjunction with an undergraduate course in magnetic design. The course has recently been reintroduced as a technical elective course where students learn the basics of inductor and transformer design. Five new laboratory experiments have been developed to cover magnetic concepts and to provide students with the hands-on experience in designing and building magnetic components. The paper presents an overview of the course, and highlights the new lab experiments in the course.
\end{abstract}

\section{INTRODUCTION}

Magnetic components have been used extensively in the practice of electrical engineering, yet courses that deal with magnetic components in any comprehensive manner are not commonly offered in universities in the U.S. One example of a widely used magnetic component in engineering systems is the inductor. Despite their common use, inductors are typically covered in undergraduate electrical engineering curriculum with emphasis on their functionalities in circuits. Hence, electrical engineering students typically possess the skill to determine the proper rating and size of inductor given a particular circuit application. However, the expectation from industry for electrical engineering graduates related to skills and knowledge on inductors or magnetics in general has increased recently to include the design aspect of magnetic components. This is due mostly to the emergence of modern applications in power systems as well as advances in power electronics for energy efficiency and renewable energy applications. Electrical engineering graduates going into power semiconductor industries are now expected to know some design skill on laminated iron-core inductors as they are widely used in the power electronics industry [1].

The importance of introducing a more comprehensive coverage of applied magnetics is further stressed by the growing list of new permanent magnet materials that promise to open up new applications that require extremely high coercivity. Examples are rare-earth alloys and amorphous metallic alloys which have now been added to the growing list of commercial magnetic materials that are available to engineers.

The issue of lack of "magneticians" has in fact long been recognized by industrial groups [2] and has encouraged several universities in the U.S. to introduce more applied magnetic design into their undergraduate curricula [3]. In an attempt to address this issue, the Education Committee of the IEEE Magnetics Society has debated the question of whether to ask for a minimum competency in magnetic design for accreditation [4], but fell short of actually taking such a step.

At Cal Poly State University in San Luis Obispo we have taken the position that magnetic design is a critical design skill set for electrical engineers. For this reason, Cal Poly has had a technical elective course in magnetic design for some time [5]. This paper focuses on the laboratory component of the course which has recently been developed to enhance students' practical skills in designing and building inductors and transformers.

\section{THE COURSE}

In 2008, the magnetic design course at Cal Poly State University underwent a major revision to cover more applied topics and to introduce new laboratory experiments where students conduct hands-on and practical designs of inductors and transformers. The course is numbered EE 433 entitled "Introduction to Magnetic Design" with the description as follows [5].

Design of magnetic components. Fundamentals of magnetics, magnetic cores, design of power transformer, three-phase transformer, dc inductor, ac inductors, dc-dc converter transformer design. Use of commercially available software. 3 lectures, 1 laboratory.

As described in the above course description, students taking the course must enroll in the laboratory portion of the course. The lab entails weekly three-hour lab time where students conduct experiments involving design calculations, 
computer simulations, and hardware tests. The software used for the simulation is LTSpice available for free from Linear Technology [6]. At the conclusion of every experiment, students must submit a report.

Five new laboratory experiments have recently been developed which aim to enforce students' understanding of inductor and transformer design. The titles of the experiments are listed below:

- Experiment \#1: Rudimentary Short Rise-Time Dynamical Reluctance Basics

- Experiment \#2: Characterizing Core Material (B-H)

- Experiment \#3: Inductor Design

- Experiment \#4: Transformer Design

- Experiment \#5: Flyback Transformer Design

The following section details each experiment.

\section{THE NEW LAB EXPERIMENTS}

\section{A. Experiment \#1}

In this experiment, the objective is to introduce students to basic hands-on magnetics construction. In addition, students investigate inductance and the energy created from loops of wire, culminating in an air-core inductor as well as effects of adding a core. In particular, students make their own inductor by winding multiple layered coils around a plastic tube that is then used to provide the energy to launch a mass (a piece of steel) placed inside the inductor.

Before conducting the hardware component, students must simulate the circuit along with the inductor that they are designing. This is done in order to determine how much inductance is needed to acquire a certain peak inductor current that relates to the inductor's stored energy.

Once the inductance value is determined, students calculate the required number of turns of the inductor. Once completed, students measure the inductance to verify whether it is in agreement with their calculation. This is done with and without the steel mass placed inside the inductor. Also, to make use of the energy stored in the inductor, students place their inductor as a component in a pre-made RLC circuit. This circuit incorporates a thyristor as a switch to transfer energy from capacitor to the inductor (Figure 1).

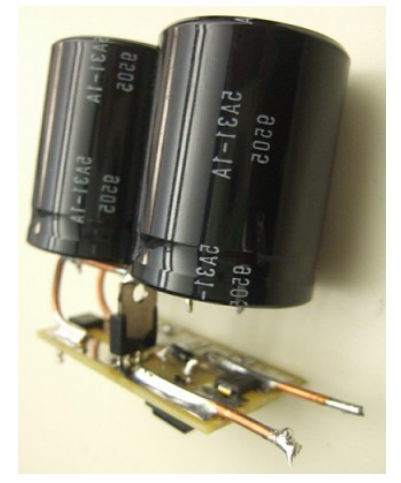

Figure 1. RLC circuit used for Experiment \#1
Once connected onto the circuit, students estimate the amount of energy being transferred from the inductor to move the mass, and perform several measurements to see if the results are in agreement with their simulated values.

\section{B. Experiment \#2}

The purpose of this experiment is for students to explore and empirically measure a core's B-H loop, saturation, and permeability. Understanding the B-H loop of a magnetic core is an important step in designing magnetic components since it shows the relationships among magnetic properties such as permeability, flux density, and magnetic field strength.

As in experiment $\# 1$, students also must run computer simulations of the circuit used to obtain the BH loop of a magnetic core. The circuit employs an inverting amplifier to amplify the small voltage dropped across a small current sense resistor which translates into the inductor current. Another op-amp is being used in the same circuit. This op-amp has been configured as an inverting integrator. Its function is to integrate the voltage across the inductor and output a signal proportional to the applied volt-seconds since volt-seconds are proportional to B. From simulation, students obtain the waveforms for inductor current and voltage before and at saturation regions of the core.

In the hardware part, students build the inductor using a given type of core. The inductor is then connected in the premade $\mathrm{BH}$ test circuit provided to students (Figure 2). The $\mathrm{BH}$ loop and inductor current and voltage are then observed from the oscilloscope. An example of saturated $\mathrm{BH}$ loop and inductor current is shown in Figs. 3 and 4.

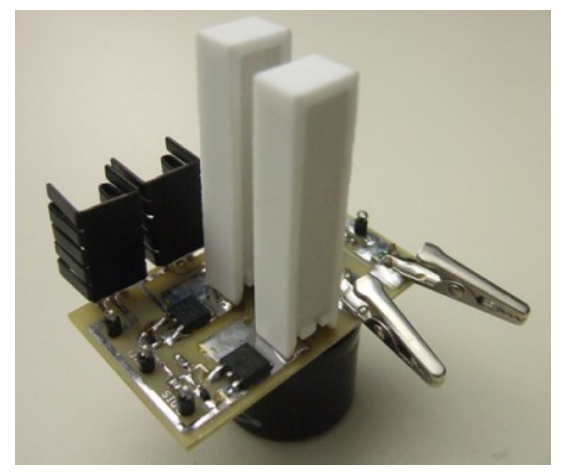

Figure 2. BH test circuit for Experiment \#2

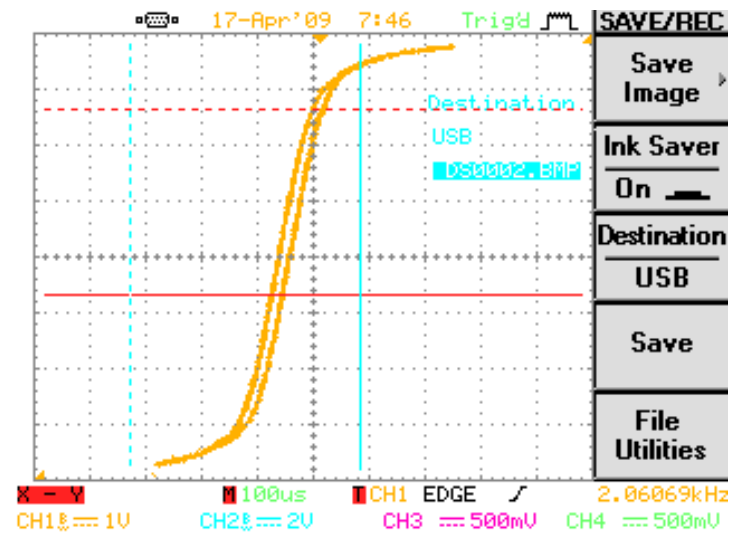

Figure 3. Saturated BH loop hardware measurement 


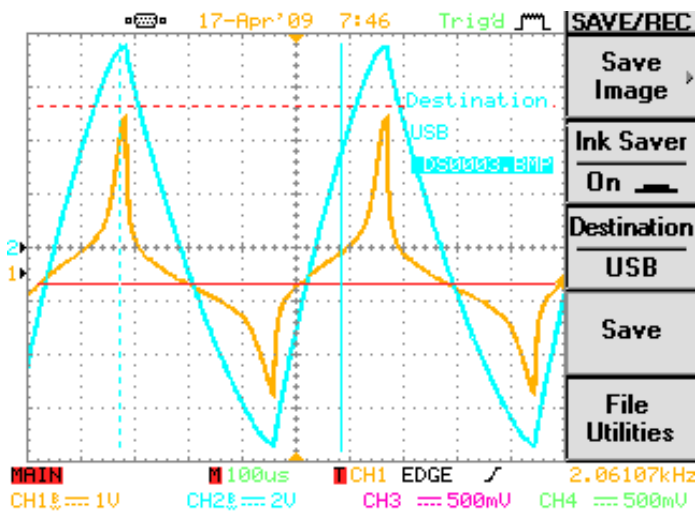

Figure 4. Saturated inductor current hardware measurement

\section{Experiment \#3}

In this experiment, students design and build an inductor for a step-down (Buck) DC-DC converter. The approach to this laboratory exercise is similar to a workplace environment, where students use available resources and consultation to aid them in the problem-solving practice. As in most real-world problems, there exist many possible solutions. Students are asked to investigate three core geometries by utilizing spreadsheet calculations to generate multiple design solutions. They then determine the viability of each design realizing that there are many trade-offs to consider. Assortment of cores (ETDs, RMs, and PQs) are made available for students to choose.

Upon completion of calculations for three selected cores, students perform computer simulation using LTSpice to confirm their design and to ensure that their chosen cores will not be saturating at given operating conditions of the buck converter. Based on these simulation results, students will have to choose one out of the three cores they selected which they think will yield the best result for the buck converter in terms of efficiency, peak to peak ripple, etc.

Hardware test follows after the decision for the ultimate core has been made. Students build their inductor and then measure the inductance to verify that they achieve the desired inductance. The inductor is then connected in a pre-made Buck converter circuit as shown in Fig. 5. Students then observe several waveforms under varying operating conditions of the converter to verify their design. An example of actual measurement of an inductor current is shown in Fig. 6.

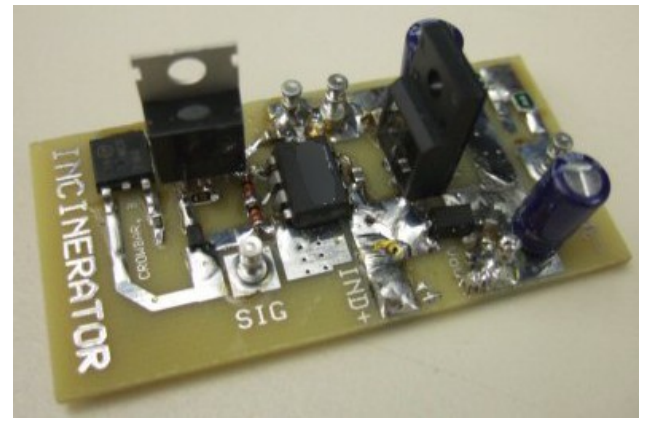

Figure 5. Buck converter test circuit for Experiment \#3

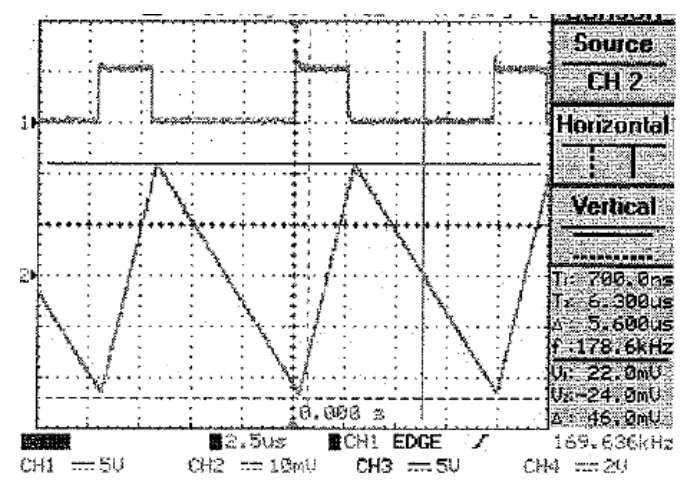

Figure 6. Inductor current hardware measurement

\section{Experiment $\# 4$}

Experiment \#4 entails the design, simulation, and construction of a transformer. In particular, the transformer is being designed for one of the isolated topologies used in power supplies called the two-switched forward converter. The topology employs two switches such as MOSFETs which are controlled synchronously to transfer energy from the source to the load through a transformer. In this experiment, students will design and build this transformer whose turnratio is related to the input voltage, output voltage and duty cycle of the converter.

As in the previous three experiments, in this lab, students start with design calculations followed by computer simulation and hardware testing. Once students verify that their transformer design calculations are comparable with the results of simulations, they will construct the transformer for hardware testing using the pre-made circuit shown in Fig. 7.

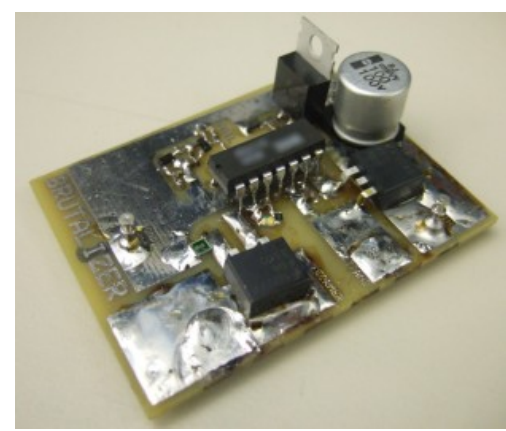

Figure 7. Transformer test circuit for Experiment \#4

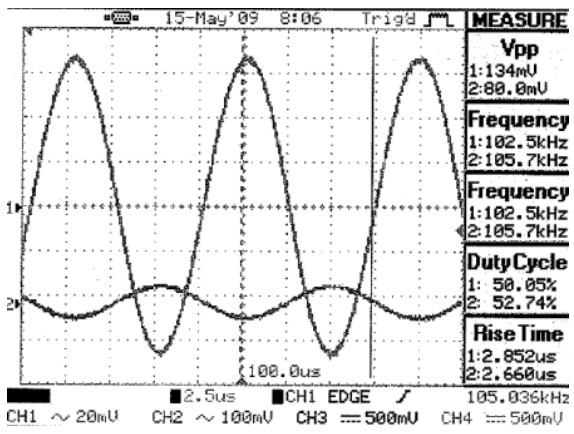

Figure 8. Inductor current hardware measurement 


\section{E. Experiment $\# 5$}

The last experiment for the course deals with the design and construction of a flyback transformer. Due to the simplicity and low parts count of a flyback converter, it has become a popular choice for low to medium power DC-DC converters in almost all industries. The flyback transformer must be designed for use in a fixed-frequency flyback circuit with the following specifications:

- Input Voltage: Vin =4.2 Battery

- Output Voltage: Vout $=48 \mathrm{~V}$

- Maximum Output Power: Pout $=10 \mathrm{~W}$

- Switching Frequency: Fs $=200 \mathrm{kHz}$

- Efficiency $>75 \%$

As in the other experiments, the test circuit (in this case, the flyback circuit) is pre-made for students to use as shown in Fig. 9. An example of winding currents is shown in Fig. 10.

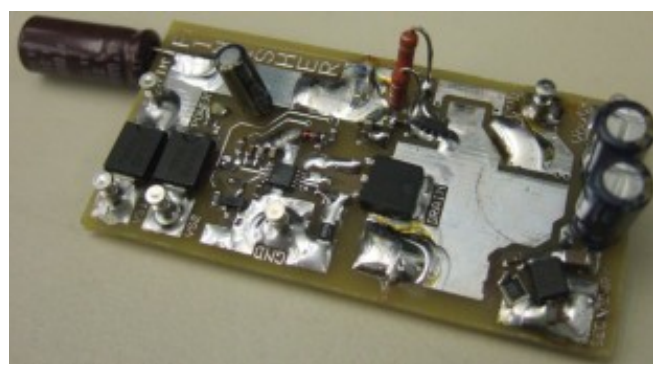

Figure 9. Inductor current hardware measurement

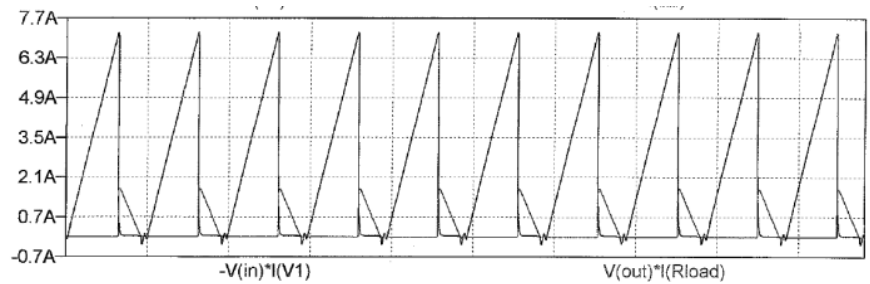

Figure 10. Primary and secondary winding currents

\section{CONCLUSIONS}

Magnetic components are undoubtedly important in many electrical circuits and in power electronic converters such as dc-dc converters. This paper presents five new laboratory experiments which have been designed in conjunction with the technical elective course in "Introduction to Magnetic Design" to prepare students for magnetics design and with the practical skills in constructing magnetic components. In these experiments, students go through the design process from calculations, computer simulations, and hardware verifications. LTSpice was chosen for the computer simulation since it is available for free and it allows user to model nonlinear characteristic of magnetic components.

The experiments were designed such that standard lab equipment (oscilloscopes, multimeters, and power supplies) may be used for measurements of results. However, to run these experiments, several different types of cores and their associated accessories (bobbins and clips) as well as magnet wires of several AWGs have to be purchased. In addition, test circuits will also have to be boarded before the course can be offered. Hence, total component cost to setup this lab could range between $\$ 2 \mathrm{k}$ and $\$ 3 \mathrm{k}$. To minimize the operational cost to run this lab, students are asked to return their completed magnetic components once they are done with the experiments.

Experience/observation from teaching the lab and students' evaluations suggest that the newly developed lab experiments are successful in enhancing students' skills and understanding of magnetic component design. Students' survey to demonstrate the effectiveness of the lab experiments in meeting their goals is planned the next time the course is offered.

\section{REFERENCES}

[1]. G. Grandi, M. K. Kazimierczuk, A. Massarini, U. Reggiani, and G. Sancineto, "Model of Laminated Iron-Core Inductors for High Frequencies", IEEE Trans. On Magnetics, Vol. 40, No. 4, July 2004.

[2]. S. Zwass, Los Angeles Chapter, IEEE Magnetics Society, letter, November 3, 1981.

[3]. J. K. Watson, "An Undergraduate Course in Applied Magnetics", IEEE Trans. On Education, Vol. E-26, No. 4, November 1983

[4]. IEEE Magnetics Society, Meet. MMM Conference, Atlanta, GA, November 11, 1981.

[5]. Electrical Engr Course Description, Cal Poly State University Website, http://www.catalog.calpoly.edu/2009pubcat/cengr/ee_dept/eecrs2009.p df, retrieved January 2011.

[6]. LT Website, http://www.linear.com/designtools/software/\#LTspice, retrieved January 2011. 